PROCEEDINGS OF THE

AMERICAN MATHEMATICAL SOCIETY

Volume 125, Number 3, March 1997, Pages 629-634

S 0002-9939(97)03581-8

\title{
GROUP ALGEBRAS WHOSE UNITS SATISFY A GROUP IDENTITY
}

\author{
ANTONIO GIAMBRUNO, SUDARSHAN SEHGAL, AND ANGELA VALENTI
}

(Communicated by Ronald M. Solomon)

\begin{abstract}
Let $F G$ be the group algebra of a torsion group over an infinite field $F$. Let $U$ be the group of units of $F G$. We prove that if $U$ satisfies a group identity, then $F G$ satisfies a polynomial identity. This confirms a conjecture of Brian Hartley.
\end{abstract}

\section{INTRODUCTION}

The unit group $U=U(F G)$ is said to satisfy a group identity if there exists a nontrivial word $w\left(x_{1}, \ldots, x_{m}\right)$, in the free group generated by $x_{1}, \ldots, x_{m}$, such that $w\left(u_{1}, \ldots, u_{m}\right)=1$ for all $u_{i} \in U$. Hartley suggested the following

Conjecture 1.1. If $G$ is a torsion group and $U(F G)$ satisfies a group identity, then FG satisfies a polynomial identity.

We recall that $F G$ is said to satisfy a polynomial identity $(P I)$ if there exists a nonzero polynomial $f\left(y_{1}, \ldots, y_{n}\right) \in F\left\{y_{1}, \ldots, y_{n}\right\}$, in noncommuting variables such that $f\left(\alpha_{1}, \ldots, \alpha_{n}\right)=0$ for all $\alpha_{i} \in F G$. Group algebras satisfying a $P I$ were classified by Passman and Isaacs-Passman.

This conjecture was first studied by Warhurst [10] who investigated special words satisfied by $U(F G)$. Pere Menal [5] suggested a possible solution for some $p$-groups. We are able to use his construction. Giambruno-Jespers-Valenti [4] settled the case where $G$ has no $p$-element if $p$ is the characteristic of $F$. Further GoncalvesMandel [3] and Dokuchaev-Goncalves [1] studied group algebras when $U$ satisfies a semigroup identity. There have been many papers classifying groups so that $U$ satisfied a special group identity like $\left(u_{1}, \ldots, u_{n}\right)$ or $\left(u^{n}, v\right)$ and many others. The main result of this paper is the following theorem.

Theorem. Suppose that $F$ is an infinite field and that $G$ is a torsion group. If $U(F G)$ satisfies a group identity, then $F G$ satisfies a polynomial identity.

\section{Some Lemmas}

We write $(u, v)=u^{-1} v^{-1} u v$ for the multiplicative commutator and $[x, y]=$ $x y-y x$ for the additive commutator. We denote by $\Delta(G, N)$ the kernel of $F G \rightarrow$

Received by the editors June 26, 1995.

1991 Mathematics Subject Classification. Primary 16S34; Secondary 20 C05.

Research supported by NR and MURST of Italy and NSERC of Canada.

(C)1997 American Mathematical Society 
$F(G / N)$ and $\Delta(G, G)=\Delta(G)$ is the augmentation ideal. Furthermore, let

$$
\begin{aligned}
& P=\left\{g \in G: o(g)=p^{k} \text { for some } k\right\}, \text { the } p \text {-elements, } \\
& Q=\{g \in G: p \nmid o(g)\}, \text { the } p^{\prime} \text {-elements, }
\end{aligned}
$$

where $p=\operatorname{char} F$. If $p=0$, we let $P=\{1\}, G=Q$. We set

$$
\phi=\phi(G)=\{g \in G: g \text { has a finite number of conjugates in } G\}
$$

to be the $F C$-subgroup of $G$, whereas

$$
\phi_{p}=\phi_{p}(G)=\langle P \cap \phi\rangle .
$$

We first record a couple of useful results and then observe what it means for a finite group $G$ that $U(F G)$ satisfies a group identity.

Lemma 2.1. Let $R$ be a semiprime ring and let $S=\{a \in R$ : for all $b, c \in R$, $b c=0$ implies $b a c=0\}$. If $S$ contains all square-zero elements of $R$, then $S$ contains all nilpotent elements of $R$.

Proof. We shall prove that $S$ contains all nilpotent elements of $R$ by induction on the index of nilpotence. Let $a \in R$ be such that $a^{n}=0, a^{n-1} \neq 0$ and suppose by induction that $x \in R, x^{m}=0$ with $m<n$ implies $x \in S$. Let $b, c \in R$ be such that $b c=0$ and let $r \in R$. Then, since $b(1-a)^{-1}(1-a) c=0$ and $(c r b)^{2}=0$, we get by hypothesis that $b(1-a)^{-1} \operatorname{crb}(1-a) c=0$. Thus

$$
b\left(1+a+a^{2}+\cdots+a^{n-1}\right) \operatorname{crb}(1-a) c=0 .
$$

Now, by the inductive hypotheses $a^{2}, a^{3}, \ldots, a^{n-1} \in S$ and we get

$$
b\left(1+a+\cdots+a^{n-1}\right) c=b a c \quad \text { and } \quad b(1-a) c=-b a c .
$$

Thus bacrbac $=0$ for all $r \in R$. Since $R$ is semiprime, then $b a c=0$ and $a \in S$.

Corollary 2.2. Let $R$ be a semiprime algebra over an infinite commutative domain $C$. If $U(R)$ satisfies a group identity, then every idempotent of $C^{-1} R$ is central and for all $b, c \in R$ such that $b c=0$ we have bac $=0$ whenever $a$ is any nilpotent element of $R$.

Proof. Lemma 2.1 and Lemma 2 of [4].

Recall that a group is called $p$-abelian if its derived group is a finite $p$-group.

Lemma 2.3. Let $G$ be a finite nonabelian group. Then $U$ satisfies a group identity if and only if $G$ is p-abelian and char $F=p$; in this case, $U$ satisfies the identity $(u, v)^{p^{n}}=1$ for some $n$ and $F G$ satisfies the polynomial identity $[x, y]^{p^{m}}=0$ for some $m$.

Proof. Let $J$ be the Jacobson radical of $F G$. Then $J$ is nilpotent and we have an epimorphism $F G \rightarrow F G / J$ which induces a homomorphism

$$
U(F G) \rightarrow U(F G / J)=\bar{U} .
$$

The latter map is onto as $u v=1-j, j \in J$, implies that $j^{k}=0$ and

$$
u v\left(1+j+j^{2}+\cdots+j^{k-1}\right)=1 .
$$

Since $U$ satisfies a group identity, so does $\bar{U}$. Now $F G / J$, being semisimple, is a direct sum of matrix rings over division rings:

$$
F G / J=\sum^{\oplus}(D)_{n \times n}
$$


Since $F G / J$ is semiprime, by 2.2 all idempotents are central; hence in the decomposition above, $n$ cannot be bigger than 1 .

Moreover, $D$ must be commutative because otherwise it contains a noncyclic free group as observed by Goncalves [2]. To see this one applies the

Tits' alternative. Any linear group is solvable by locally finite or it contains a noncyclic free group.

We have, therefore, seen that $F G / J$ is commutative. It follows that $(x, y)=$ $1 \bmod J$ for all $x, y \in G$ and consequently $G^{\prime} \subseteq 1+J$. Thus $\Delta\left(G^{\prime}\right) \subseteq J$ is nilpotent and $G^{\prime}$ is a $p$-group with $p=\operatorname{char} F$ as asserted.

Now, suppose that $G^{\prime}$ is a (finite) $p$-group so that $\left(\Delta G^{\prime}\right)^{p^{k}}=0$ for some $k$. Then for all $u, v \in F G$ we have $(u, v)=1 \bmod \Delta\left(G, G^{\prime}\right)$ and $(u, v)^{p^{k}} \in 1+\left(\Delta\left(G, G^{\prime}\right)\right)^{p^{k}}=$ $1+F G\left(\Delta G^{\prime}\right)^{p^{k}}=1$. Moreover, for $x, y \in F G$

$$
\begin{aligned}
{[x, y] } & =\sum c[g, h] \quad(c \in F, g, h \in G) \\
& =\sum c g h(1-(g, h)) \in \Delta\left(G, G^{\prime}\right) .
\end{aligned}
$$

It follows that $[x, y]^{p^{k}}=0$. This completes the proof of the lemma.

We shall need the following classical results.

Lemma 2.4. (a) (Isaacs-Passman) If char $K=0$, then $K G$ satisfies $a P I$ if and only if $G$ contains an abelian subgroup of finite index.

(b) (Passman) If char $K=p>0$, then $K G$ satisfies a PI if and only if $G$ contains a p-abelian subgroup of finite index.

Proof. See [6, p. 196].

Lemma 2.5. (Passman) Suppose that char $K=p>0$. Then $K G$ is semiprime if and only if $\phi(G)$ is a $p^{\prime}$-group.

Proof. See [6, p. 131].

Let $N=N(K G)$ be the sum of all nilpotent ideals of $K G$.

Lemma 2.6. (Passman) Suppose that char $K=p>0$. Then $N(K G)$ is nilpotent if and only if $\phi_{p}(G)$ is finite.

Proof. See [6, p. 311].

\section{Proof of the Theorem}

We shall divide the proof into the following three exhaustive and mutually exclusive cases

(i) $F G$ is semiprime, i.e. $N=0$.

(ii) $N$ is (nonzero) nilpotent.

(iii) $N$ is nil but not nilpotent.

Case (i): $F G$ semiprime.

Let $y \in Q$ have order $m, p \nmid m$. Write

$$
\hat{y}=1+y+\cdots+y^{m-1} .
$$

Then $\hat{y} / m=e=e^{2}$ is central by 2.2. It follows that $\langle y\rangle$ is normal in $G$. Thus $Q$ is abelian or Hamiltonian, namely, $K_{8} \times A$ where $K_{8}$ is the quaternion group of order 
8 and $A$ is abelian. But $K_{8}$ cannot arise as $F K_{8}$ will have a summand which is a division ring or a ring of matrices, both not allowed as seen in 2.3. In any case, $Q$ is abelian. If char $F=0$, then $Q=G$ is abelian. Thus we may suppose that $p>0$.

Let $g, h \in P$. Then $(1-g)$ is nilpotent and $\hat{h}(1-h)=0$. Therefore, by 2.2 , we have

$$
0=\hat{h}(1-g)(1-h)=\hat{h} g(1-h) .
$$

It follows that $\hat{h} g h=\hat{h} g$ and, therefore, $g=h^{i} g h$ for some $i$. Thus $g h g^{-1}=h^{-i}$ and $\langle h\rangle \triangleleft P$. We have proved that $P$ is a group which is abelian or Hamiltonian. We shall see, in a moment, that $P$ will turn out to be trivial.

Take $\pi \in P$ and let $g \in Q$. Since $\langle g\rangle \triangleleft G$, it follows that $H=\langle g, \pi\rangle$ is a finite subgroup of $G$. By Lemma 2.3, $H$ is $p$-abelian, so the commutator $(g, \pi)$ is a $p$ element in $\langle g\rangle$. Hence $(g, \pi)=1$ and it follows that $\langle\pi\rangle$ is normalized by $Q$ and also by $P$. Thus $\langle\pi\rangle \triangleleft G$ and, since $F G$ is semiprime, we have $\pi=1$ and $G=Q$ is abelian.

Case (ii): $N$ (nonzero) nilpotent.

Since $N$ is nilpotent, $\phi_{p}(G)$ is a finite group by 2.6 and $p=\operatorname{char} F$. Then $\phi\left(G / \phi_{p}(G)\right)$ has no $p$-elements. So $F\left(G / \phi_{p}(G)\right)$ is semiprime.

To the finite group $\phi_{p}(G)$ we apply 2.3 to conclude that its commutator group is a $p$-group. It follows that the $p$-elements of $\phi_{p}(G)$ form a group. Since $\phi_{p}(G)$ is generated by $p$-elements it is a $p$-group.

Thus we have an epimorphism $U(F G) \rightarrow U F\left(G / \phi_{p}\right)=\bar{U}$. Since $\bar{U}$ satisfies a group identity and $F\left(G / \phi_{p}\right)$ is semiprime we can apply case (i) to conclude that $G / \phi_{p}$ is abelian. Thus $G^{\prime}$ is contained in $\phi_{p}$ and is a finite $p$-group. We have proved that $F G$ satisfies a polynomial identity (of the form $[x, y]^{p^{m}}=0$ ).

Case (iii): $N$ nil but not nilpotent.

Let $R=F\{X\}$ be the free algebra on a countable set $X=\left\{x_{1}, x_{2}, \ldots\right\}$. Let $t$ be an indeterminate and $R[[t]]$ the power series ring over $R$. Finally, let $w=w\left(y_{1}, \ldots, y_{m}\right)$ be the nontrivial group identity satisfied by $U(F G)$. Then the elements $1+x_{1} t, 1+x_{2} t, \ldots, 1+x_{n} t$ are units in $R[[t]]$. They generate a free group by the Magnus argument:

Suppose

$$
\left(1+x_{i_{1}} t\right)^{\alpha_{1}}\left(1+x_{i_{2}} t\right)^{\alpha_{2}} \cdots\left(1+x_{i_{e}} t\right)^{\alpha_{e}}=1, \quad \alpha_{i} \neq 0 .
$$

Write $\alpha_{i}=p^{s_{i}} \beta_{i}, p \nmid \beta_{i}$. Then

$$
\left(1+y_{i_{1}} t^{p^{s_{1}}}\right)^{\beta_{1}}\left(1+y_{i_{2}} t^{p^{s_{2}}}\right)^{\beta_{2}} \cdots\left(1+y_{i_{e}} t^{p^{s_{e}}}\right)^{\beta_{e}}=1 \text { with } y_{i_{k}}=x_{i_{k}}^{p^{s_{k}}} .
$$

We observe that the coefficient of $y_{i_{1}} y_{i_{2}} \cdots y_{i_{e}} t^{\Sigma p^{s_{j}}}$ on the left-hand side is $\beta_{1} \beta_{2} \cdots$ $\beta_{s}$, which is not zero, giving us a contradiction.

Substituting $1+x_{i} t$ for $y_{i}$ in $w\left(y_{1}, \ldots, y_{m}\right)$ we get an expression

$$
1 \neq\left(1+x_{i_{1}} t\right)^{l_{1}}\left(1+x_{i_{2}} t\right)^{l_{2}} \cdots\left(1+x_{i_{s}}\right)^{l_{s}} \in R[[t]] .
$$

This can be rewritten in the form

$$
\sum_{i \geq 1} p_{i}\left(x_{1}, \ldots, x_{m}\right) t^{i} \neq 0
$$

Thus there exists $l \geq 1$ such that $p_{l}\left(x_{1}, \ldots, x_{m}\right) \neq 0$ and $p_{l}\left(x_{1}, \ldots, x_{m}\right) \in R=$ $F\left\{x_{1}, \ldots, x_{m}\right\}$ is a homogeneous polynomial of degree $l$. 
Now, if $r_{1}, \ldots, r_{m} \in N(F G)$, the elements $1+r_{i} \lambda$ are invertible in $F G$ with inverse

$$
\left(1+r_{i} \lambda\right)^{-1}=\left(1-r_{i} \lambda+r_{i}^{2} \lambda^{2}-\cdots\right) .
$$

Hence, by evaluating the group identity on the elements $1+r_{1} \lambda, \ldots, 1+r_{m} \lambda$, we get

$$
\sum_{i=1}^{k} p_{i}\left(r_{1}, \ldots, r_{m}\right) \lambda^{i}=0
$$

for some positive integer $k$ and $p_{t}\left(r_{1}, \ldots, r_{m}\right)=0$ for all $t>k$. Hence, if $l>k$, then $p_{l}\left(r_{1}, \ldots, r_{m}\right)=0$. On the other hand if $l \leq k$, since $F$ is infinite there exist nonzero distinct elements $\lambda_{1}, \ldots, \lambda_{k+1} \in F$ such that $\sum_{i=1}^{k} p_{i}\left(r_{1}, \ldots, r_{m}\right) \lambda_{j}^{i}=0$, for all $j=1, \ldots, k+1$. But then

$$
\left(\begin{array}{cccc}
1 & \lambda_{1} & \ldots & \lambda_{1}^{k} \\
1 & \lambda_{2} & \ldots & \lambda_{2}^{k} \\
\vdots & \vdots & & \vdots \\
1 & \lambda_{k+1} & \ldots & \lambda_{k+1}^{k}
\end{array}\right)\left(\begin{array}{c}
0 \\
p_{1}\left(r_{1}, \ldots, r_{m}\right) \\
\vdots \\
p_{k}\left(r_{1}, \ldots, r_{m}\right)
\end{array}\right)=0
$$

and, since the determinant of the above Vandermonde matrix is nonzero, we get

$$
p_{1}\left(r_{1}, \ldots, r_{m}\right)=\cdots=p_{k}\left(r_{1}, \ldots, r_{m}\right)=0 \text {. }
$$

Thus $p_{l}\left(r_{1}, \ldots, r_{m}\right)=0$ also in this case. We have proved that $p_{l}\left(x_{1}, \ldots, x_{m}\right)$ is a $P I$ for $N(F G)$.

By a standard linearization process it follows that $N(F G)$ satisfies a multilinear polynomial identity:

$$
f\left(x_{1}, \ldots, x_{d}\right)=\sum_{\sigma \in S_{d}} \alpha_{\sigma} x_{\sigma(1)} \cdots x_{\sigma(d)}
$$

where $\alpha_{\sigma} \in F$ and $S_{d}$ is the symmetric group of degree $d$. Since $N(F G)$ is not nilpotent we can choose $a_{1}, \ldots, a_{d} \in N(F G)$ so that $a_{1} a_{2} \cdots a_{d} \neq 0$. Then

$$
a_{1} F G a_{2} F G \cdots a_{d} F G \neq 0
$$

and

$$
\sum_{\sigma \in S_{d}} \alpha_{\sigma} a_{\sigma(1)} x_{\sigma(1)} a_{\sigma(2)} x_{\sigma(2)} \cdots a_{\sigma(d)} x_{\sigma(d)}
$$

is a nondegenerate multilinear generalized polynomial identity for $F G$. By a theorem of Passman [6, p. 202] we conclude that $(G: \phi)<\infty$ and $\left(\phi^{\prime}: 1\right)<\infty$. It remains to prove that $\phi^{\prime}$ is a $p$-group. This follows because now $G$ is locally finite and we can apply 2.3 .

Corollary. Let $F$ be an infinite field and let $G$ be a torsion nilpotent group which is nonabelian. Then $U(F G)$ satisfies a group identity $\Leftrightarrow F G$ is Lie $n$-Engel $\Leftrightarrow$ $\left(u^{p^{m}}, v\right)=1 \forall u, v \in U$ and some fixed $m, p=\operatorname{char} F$.

Proof. Suppose $U$ satisfies a group identity. Then by the theorem we have $(G: \phi)<\infty,\left(\phi^{\prime}: 1\right)<\infty$. Remembering that $G=P \times Q$ is locally finite, we conclude by 2.3 that $G^{\prime} \subseteq P$. Thus $Q$ is central and $Q \subseteq \phi$. Thus $(G: \phi)$ is a $p$-power. Moreover, $\left(\phi^{\prime}: 1\right)$ is a $p$-power. It follows by a theorem of Sehgal [8, p. $155]$ that $F G$ is Lie $n$-Engel. 
Suppose that $F G$ is Lie $n$-Engel. Then $[x, \underbrace{y, \ldots, y}_{n}]=0$. Choosing $m$ so that $p^{m}>n$, we have $0=[x, \underbrace{y, \ldots, y}_{p^{m}}]=\left[x, y^{p^{m}}\right]$ which implies $\left(u^{p^{m}}, v\right)=1$ for $u, v \in U$.

The remaining implication is trivial. This completes the proof of the Corollary.

\section{ACKNOWLEDGMENT}

We are thankful to Don Passman for helpful suggestions.

\section{REFERENCES}

1. M. Dokuchaev and J. Z. Goncalves, Semigroup identities on units of integral group rings, Glasgow Math. J. (to appear).

2. J. Z. Goncalves, Free subgroups of units in group rings, Canad. Math. Bull. 27 (1984), 309312. MR 85k:20021

3. J. Z. Goncalves and A. Mandel, Semigroup identities on units of group algebras, Arch. Math. 57 (1991), 539-545. MR 93g:16049

4. A. Giambruno, E. Jespers and A. Valenti, Group identities on units of rings, Arch. Math. 63 (1994), 241-296. MR 95h:16044

5. P. Menal, Private letter to B. Hartley, April 6, 1981.

6. D. S. Passman, The Algebraic Structure of Group Rings, John Wiley, New York, 1977. MR 81d:16001

7. I. Reiner, Maximal Orders, Academic Press, New York, 1975. MR 52:13910

8. S. K. Sehgal, Topics in Group Rings, Marcel Dekker, New York, 1978. MR 80j:16001

9. __ Units in Integral Group Rings, Longman, Essex, 1993. MR 94m:16039

10. D. S. Warhurst, Topics in Group Rings, Thesis, Manchester, 1981.

(A. Giambruno and A. Valenti) Dipartimento di Matematica, Universitá di Palermo, via Archirafi 34, 90123 Palermo, Italy

E-mail address: giambruno@ipamat.math.unipa.it

E-mail address: avalenti@ipamat.math.unipa.it

(S. Sehgal) Department of Mathematical Sciences, University of Alberta, Edmonton, Alberta, Canada T6G 2G1

E-mail address: ssehgal@schur.math.ualberta.ca 